\title{
c-Abl-p38a signaling plays an important role in MPTP-induced neuronal death
}

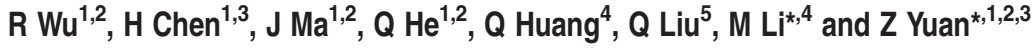

Oxidative stress is a major cause of sporadic Parkinson's disease (PD). Here, we demonstrated that c-Abl plays an important role in oxidative stress-induced neuronal cell death. C-Abl, a nonreceptor tyrosine kinase, was activated in an 1-methyl-4-phenyl-1,2,3,6tetrahydropyridine hydrochloride (MPTP)-induced acute PD model. Conditional knockout of c-Abl in neurons or treatment of mice with STI571, a c-Abl family kinase inhibitor, reduced the loss of dopaminergic neurons and ameliorated the locomotive defects induced by short-term MPTP treatment. By combining the SILAC (stable isotope labeling with amino acids in cell culture) technique with other biochemical methods, we identified $\mathrm{p} 38 \alpha$ as a major substrate of c-Abl both in vitro and in vivo and c-Ablmediated phosphorylation is critical for the dimerization of p38 $\alpha$. Furthermore, p38 $\alpha$ inhibition mitigated the MPTP-induced loss of dopaminergic neurons. Taken together, these data suggested that c-Abl-p38 $\alpha$ signaling may represent a therapeutic target for PD. Cell Death and Differentiation (2016) 23, 542-552; doi:10.1038/cdd.2015.135; published online 30 October 2015

Parkinson's disease (PD), the second most common neurodegenerative disorder, is characterized by bradykinesia, rigidity, tremor, and loss of dopaminergic neurons. ${ }^{1}$ Familial mutations that cause PD have been identified, including in the genes that encode $a$-synuclein and leucine-rich repeat kinase 2 (LRRK2) that cause autosomal-dominant PD, and DJ-1, PINK1, and parkin that cause autosomal-recessive PD. ${ }^{2}$ However, the majority of PD cases are sporadic. The cause of sporadic PD remains unknown, and the role of environmental toxins and genetic factors in sporadic PD is unclear. However, the evidence regarding postencephalitic PD and the discovery of 1-methyl-4-phenyl-1,2,3,6-tetrahydropyridine hydrochloride (MPTP)-induced Parkinsonism suggest that environmental toxins may be a major cause of sporadic PD. ${ }^{3,4}$

The neurotoxins used to induce dopaminergic neurodegeneration, including 6-hydroxydopamine, MPTP, and rotenone, induce the formation of reactive oxygen species (ROS). ROS react with nucleic acids, proteins, and lipids to induce mitochondrial damage. Although oxidative stress plays a critical role in causing PD, the mechanisms underlying oxidative stress-induced PD remain unclear.

The nonreceptor tyrosine kinase c-Abl is ubiquitously expressed and mediates a variety of extrinsic and intrinsic cell signaling activities, including growth factor signaling, cell adhesion, oxidative stress, and DNA damage ${ }^{5}$ Our group and other groups have reported that C-Abl plays an important role in oxidative stress-induced neuronal death. ${ }^{6-8}$ Recently, Ko et al. ${ }^{9}$ and Imam et al. ${ }^{10}$ have reported that c-Abl phosphorylated Parkin and inhibited its E3 ligase activity that led to the neurotoxic accumulation of Parkin's substrates. $a$-Synuclein has also been reported to be substrates of $\mathrm{c}-\mathrm{Abl}$ and to participate in PD pathogenesis. ${ }^{9-11}$ The c-Abl inhibitor Nilotinib and INNO-406 have been reported prevents the loss of dopamine neurons and improves motor behavior in a murine PD model. ${ }^{12-14}$

In this study, we demonstrated that c-Abl is activated in oxidative stress-induced PD. Both the conditional knockout (KO) of c-Abl and treatment with the c-Abl inhibitor STI571 protect against MPTP-induced PD. Using the SILAC (stable isotope labeling with amino acids in cell culture) technique, we showed that p38a is a novel c-Abl substrate that mediates oxidative stress-induced PD. The phosphorylation of p38a at Y182 and Y323 by c-Abl promotes p38a dimerization, thereby activating p38a via a noncanonical pathway. The inhibition of p38a using SB203580 mitigates the MPTP-induced loss of dopaminergic neurons. Taken together, we found that c-Abl-p38a signaling plays a role in oxidative stress-induced PD.

${ }^{1}$ State Key Laboratory of Brain and Cognitive Sciences, Institute of Biophysics, Chinese Academy of Sciences, Beijing 100101, China; ${ }^{2}$ College of Life Sciences, University of Chinese Academy of Sciences, Beijing 100049, China; ${ }^{3}$ Center of Alzheimer's Disease, Beijing Institute for Brain Disorders, Beijing 100069, China; ${ }^{4}$ Department of Pharmacology and the Proteomics Center, Zhongshan School of Medicine, Sun Yat-sen University, Guangzhou 510080, China and ${ }^{5}$ High Magnetic Field Laboratory, Chinese Academy of Sciences, Hefei, Anhui 230031, China

*Corresponding author: M Li, Department of Pharmacology and the Proteomics Center, Zhongshan School of Medicine, Sun Yat-sen University, Guangzhou 510080, China. Tel: +86 20 87331553; Fax: +86 20 87331653; E-mail: limt@ mail.sysu.edu.cn

or Z Yuan, State Key Laboratory of Brain and Cognitive Sciences, Institute of Biophysics, Chinese Academy of Sciences, Beijing 100101, China. Tel: +86 10 64867137; Fax: +86 10 64867137; E-mail: zqyuan@ibp.ac.cn

Abbreviations: AIMP2, aminoacyl tRNA synthetase complex-interacting multifunctional protein 2; ANOVA, one-way analysis of variance; DAB, 3,3'-diaminobenzidine; EGTA, ethylene glycol tetraacetic acid; FAK, focal adhesion kinase; FBP1, far upstream element-binding protein 1; FBS, fetal bovine serum; GST, glutathione S-transferase; HPLC, high-performance liquid chromatography; HRP, horseradish peroxidase; IP, immunoprecipitation; JNK, c-Jun N-terminal kinase; KD, kinase dead; KO, knockout; LCMS, liquid chromatography-mass spectrometry; LRRK2, leucine-rich repeat kinase 2; MAPK, mitogen-activated protein kinase; MPP+, 1-methyl-4-phenylpyridinium; MPTP, 1-methyl-4-phenyl-1,2,3,6-tetrahydropyridine hydrochloride; MST1, mammalian Ste20-like kinase 1; PD, Parkinson's disease; PINK1, PTEN-induced putative kinase 1; ROS, reactive oxygen species; SILAC, stable isotope labeling with amino acids in cell culture; TBST, Tris-buffered saline and Tween-20; TFA, trifluoroacetate; TH, tyrosine hydroxylase; TSA, tyramide signal amplification; WASP, Wiskott-Aldrich syndrome protein; WT, wild type

Received 15.4.15; revised 30.8.15; accepted 07.9.15; Edited by R Knight; published online 30.10.15 


\section{Results}

Conditional $\mathrm{KO}$ of $\mathrm{c}-\mathrm{Abl}$ in neurons protects against MPTP-induced death of dopaminergic neurons. We previously reported that $\mathrm{c}-\mathrm{AbI}$ mediates oxidative stress-induced neuronal death. ${ }^{6,7}$ Here, we investigated the role of $\mathrm{c}-\mathrm{Abl}$ in oxidative stress-induced neurodegeneration and the underlying mechanisms. C57BL/6J mice were used to assess the importance of c-Abl in the MPTP-induced model of PD. The mice were treated with either saline or MPTP (four intraperitoneal injections of $20 \mathrm{mg} / \mathrm{kg}$ at $2 \mathrm{~h}$ intervals). At $2 \mathrm{~h}$ and on each of 7 consecutive days after the final MPTP injection, c-Abl phosphorylation at Y245 was measured in the striatum to determine the level of c-Abl activation. MPTP treatment caused a 1.4-fold increase in the phospho-Y245-cAbl level from $2 \mathrm{~h}$ to 2 days after MPTP injection. We observed a dramatic decrease in the tyrosine hydroxylase (TH) protein levels 1 day after MPTP injection, followed by a mild decrease in TH expression from 3 to 7 days after MPTP injection (Figures $1 \mathrm{a}$ and $\mathrm{b}$ ). These data suggested that c-Abl activation may participate in the MPTP-induced loss of dopaminergic neurons. To confirm this result, $\mathrm{c}-\left.\mathrm{Abl}\right|^{\text {flox/flox }}$ mice were crossed with CaMKII-iCre transgenic mice to generate conditional $\mathrm{KO}$ of $\mathrm{c}-\mathrm{Abl}$ in neurons. Wild-type (WT, c-Abl ${ }^{\text {flox/flox }}$ ) and c-Abl KO (c-Abl flox/flox; CaMKII-iCre ${ }^{+/-}$) mice were treated with saline or MPTP (four intraperitoneal injections of $20 \mathrm{mg} / \mathrm{kg}$ at $2 \mathrm{~h}$ intervals). After treatment with MPTP, the loss of neurons was monitored via stereological analysis of $\mathrm{TH}$ or Nissl immunostaining in substantia nigra. MPTP induced the loss of $\sim 40 \%$ of the $\mathrm{TH}$-positive neurons (Figures 1c and d) and the similar result was observed in Nissl staining (Figure 1e). The neuron-specific KO of c-Abl resulted in significant protection against the MPTP-induced death of neurons compared with endogenous WT c-Abl expression (Figures 1c-e). The level of the $\mathrm{TH}$ protein in the striatum also indicated that $\mathrm{C}-\mathrm{Abl} \mathrm{KO}$ prevented the loss of dopaminergic neurons following MPTP exposure (Figures $1 f$ and $g$ ). a

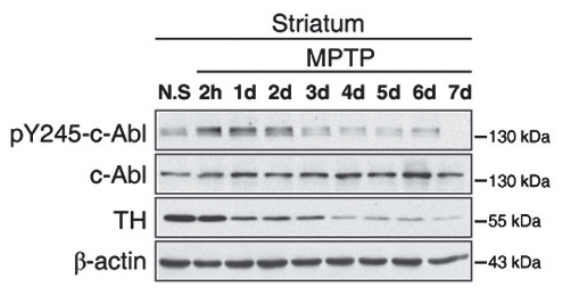

C

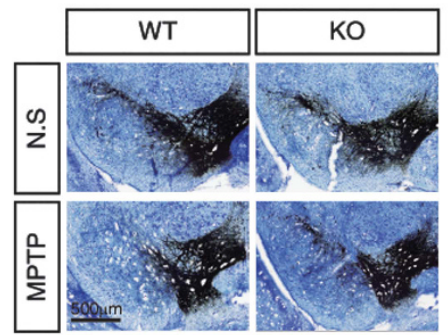

f

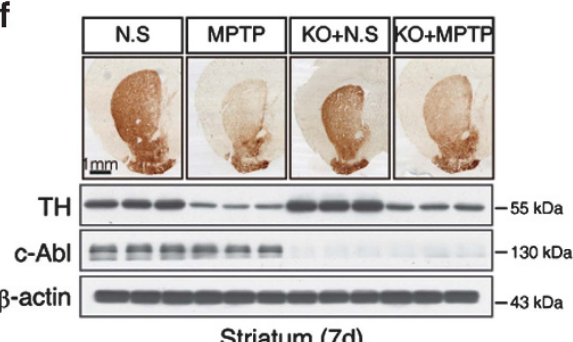

b
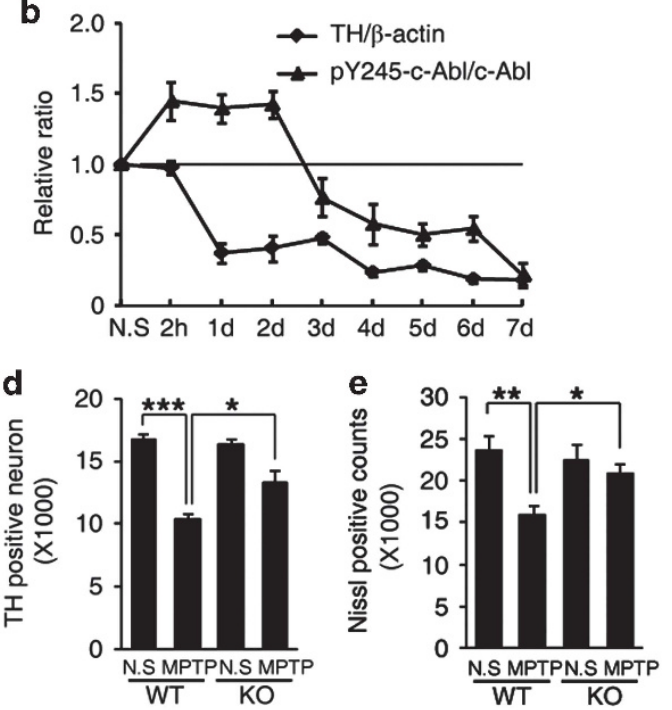

9

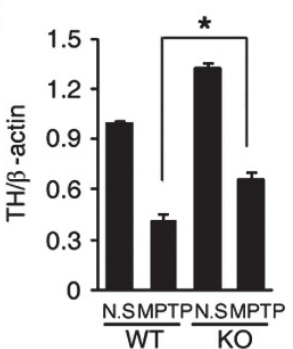

h

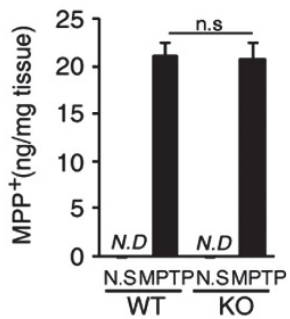

Figure $1 \mathrm{c}$-Abl is activated in an MPTP-induced PD model. (a) C57BL/6J mice were treated with saline or MPTP (four i.p. injections of $20 \mathrm{mg} / \mathrm{kg}$ at $2 \mathrm{~h}$ intervals). Striatum tissue was collected at $2 \mathrm{~h}$ and on each of 7 consecutive days after MPTP injection. The striatal tissue lysates were immunoblotted using an anti-phospho-Y245-c-Abl (pY245-c$\mathrm{Abl}$ ) antibody to determine the levels of tyrosine-phosphorylated c-Abl. An anti- $\beta$-actin antibody was used as a loading control, and a anti-TH antibody was used to label dopaminergic neurons after MPTP treatment. (b) The normalized levels of pY245-c-Abl and TH. The data are expressed as mean \pm S.E.M. (c) Photomicrographs of TH and Niss co-stained sections from the substantia nigra of WT and c-Abl KO littermate mice treated with saline or MPTP. (d and e) The number of TH-positive neurons (d) and Nissl-stained cells (e) in the substantia nigra of the WT and c-Abl KO littermate mice treated with saline or MPTP, as determined by stereological quantification. The data are expressed as mean \pm S.E.M. (ANOVA, ${ }^{\star} P<0.05,{ }^{* \star} P<0.01,{ }^{* \star \star} P<0.001, n=5$ ). (f) Photomicrographs of TH-immunostained sections from the striatum of WT and $c$-Abl KO littermate mice treated with saline or MPTP (upper panel). Striatal tissue was collected from WT and c-Abl KO littermate mice 7 days after treatment with saline or MPTP and was subjected to immunoblotting using the indicated antibodies (bottom panel). (g) The normalized levels of TH based on quantification using ImageJ software (NIH). The data are expressed as mean \pm S.E.M. (ANOVA, ${ }^{*} \mathrm{P}<0.05, n=3$ ). (h) Levels of MPP ${ }^{+}$in the striatum of WT and c-Abl KO mice treated with saline and MPTP (four i.p. injections. $20 \mathrm{mg} / \mathrm{kg}$, at $2 \mathrm{~h}$ intervals). ND, not detected 
In addition, we observed that there is similar concentration of $\mathrm{MPP}^{+}$, the active metabolite of MPTP in the brain, in WT and c-Abl KO mice (Figure 1h). Together, these data suggested that MPTP mediates the activation of $c-A b l$ and that the neuron-specific KO of c-Abl prevents MPTP-induced dopaminergic neuronal death.

STI571 reduces the loss of dopaminergic neurons and ameliorates the locomotive defects induced by acute MPTP treatment. To determine whether an inhibitor of c-Abl protects against the MPTP-induced loss of dopaminergic neurons, we treated animals with STI571, a c-Abl family kinase inhibitor, before or after exposure to MPTP (Figure 2a). It has been shown that intraperitoneally injected STI571 could penetrate into brain, ${ }^{15}$ and we confirmed the presence of STI571 in the ventral midbrain by intraperitoneal injection in our experiments (Figure 2b). Moreover, we found that intraperitoneal injection of STI571 did not affect the concentration of $\mathrm{MPP}^{+}$, the metabolite of MPTP, in the striatum (Figure 2c). Pretreating mice with STI571 prevented the MPTP-induced tyrosine phosphorylation of c-Abl (Figure 2d). At 7 days after the final MPTP injection, stereological analysis of the $\mathrm{TH}$-positive neurons and Nissl staining cell showed that STI571 markedly protected against the MPTP-induced death of neurons (Figures $2 \mathrm{e}-\mathrm{g}$ ). The expression levels of $\mathrm{TH}$ in the striatum were dramatically decreased following MPTP treatment, but STI571 treatment significantly rescued the level of $\mathrm{TH}$ (Figures $2 \mathrm{~h}$ and i). The loss of dopaminergic neurons from the substantia nigra is always accompanied by the development of motor defects. Rota-Rod tests showed that STI571 protected against MPTPinduced locomotive defects (Figure 2j). These data showed that treatment with an inhibitor of c-Abl rescues dopaminergic neurons from MPTP-induced death. Therefore, treatment with a c-Abl inhibitor may also improve motor defects in patients with PD.

SILAC identified p38a as the major substrate for c-Abl during oxidative stress. It has been reported that $c-A b l$ mediates PD pathogenesis via targets such as parkin and $a$-synuclein. ${ }^{9,10,16}$ However, the molecular mechanisms by which c-Abl participates in oxidative stress-induced PD remain unknown. Because $\mathrm{c}-\mathrm{Abl}$ is a tyrosine kinase, its substrates can be determined via SILAC technology followed by the identification of the tyrosine-phosphorylated peptides. Three populations of SH-SY5Y cells were independently cultured in the presence of 'light' arginine $\left(\operatorname{Arg0}{ }^{12} \mathrm{C}_{6}^{14} \mathrm{~N}_{4}\right)$ and lysine (Lys0 ${ }^{12} \mathrm{C}_{6}^{14} \mathrm{~N}_{2}$ ), 'medium' arginine (Arg6 ${ }^{13} \mathrm{C}_{6}^{14} \mathrm{~N}_{4}$ ) and lysine (Lys6 ${ }^{13} \mathrm{C}_{6}^{14} \mathrm{~N}_{2}$ ), or 'heavy' arginine (Arg10 ${ }^{13} \mathrm{C}_{6}^{15} \mathrm{~N}_{4}$ ) and lysine (Lys8 ${ }^{13} \mathrm{C}_{6}^{15} \mathrm{~N}_{2}$ ) (Figure 3a). The labeled SH-SY5Y cells were treated with vehicle, hydrogen peroxide alone, or hydrogen peroxide and the c-Abl inhibitor STI571. Partial cell lysates were immunoprecipitated using an anti-panphosphotyrosine antibody and then immunoblotted using an anti-c-Abl antibody. Hydrogen peroxide treatment induced c-Abl activation (Figure $3 b$ ). The labeled cells were lysed under denaturing conditions and were mixed together in equal portions. The tyrosine-phosphorylated peptides were isolated and analyzed via liquid chromatography-mass spectrometry (LC-MS).
In the list of possible substrates of c-Abl by SILAC, Arg, ${ }^{17}$ focal adhesion kinase (FAK) ${ }^{18}$ Caveolin- $1,{ }^{19}$ and WiskottAldrich syndrome protein (WASP) ${ }^{20}$ have been reported. Interestingly, a subgroup of mitogen-activated protein kinases (MAPKs) were identified in the peptide list (Figure 3c), among which MAPK14 (encoding p38a) expression was increased 3.6-fold by hydrogen peroxide treatment and was decreased by $\sim 30 \%$ by STI571 treatment. These data suggested that p38a may serve as a substrate of the kinase c-Abl under oxidative stress conditions. To confirm that p38 $a$ is a substrate of $c-A b l$, the SILAC samples were immunoprecipitated using an anti-pan-phosphotyrosine antibody and were immunoblotted using an anti-p38a antibody. The increased tyrosine phosphorylation of p38a mediated by oxidative stress was mitigated by STI571 (Figure 3d). Accordingly, in vitro kinase assays demonstrated that the kinase $\mathrm{c}$-Abl phosphorylated p38a (Figure 3e). Moreover, the c-Abl-mediated phosphorylation of p38a was observed using WT c-Abl but not kinase-dead (KD) c-Abl (Figure 3f).

c-Abl interacts with p38a and phosphorylates p38a at Y182 and Y323. To determine whether p38a directly interacts with c-Abl, a glutathione $S$-transferase (GST) pull-down assay was performed by incubating the recombinant GSTp38a with cell lysates that expressed Myc-tagged c-Abl. C-Abl interacts with GST-p38a but not GST alone (Figure 4a). Co-immunoprecipitation results showed that c-Abl interacts with p38a in cells (Figures $4 b$ and c). To further map the p38a phosphorylation sites targeted by $\mathrm{c}-\mathrm{Abl}$ kinase, in vitro phosphorylated p38a was subjected to mass spectrometry analysis. Phosphorylated tyrosine was identified at two sites, tyrosine 182 (Y182) and tyrosine 323 (Y323) (Figure 4d). In cell culture experiments, c-Abl phosphorylated p38a-WT; however, the phosphorylation of p38a-Y182F, p38a-Y323F, and p38a-Y182F/Y323F by c-Abl was dramatically reduced (Figure $4 \mathrm{e}$ ). These data suggested that $\mathrm{c}-\mathrm{Abl}$ phosphorylates p38a at Y182 and Y323. Consistently, we observed p38a autophosphorylation (T180/Y182), and STI571 treatment inhibits this phosphorylation (Figure 3d). Interestingly, the autophosphorylation of p38a at T180/Y182 was decreased when $\mathrm{Y} 323$ was replaced with a phenylalanine (Figure 4e). In addition to the well-characterized activation of $\mathrm{p} 38 a$ via phosphorylation at both residues of the p38a TxY activation loop motif by dual T/Y-specific MAPK kinases, $\mathrm{p} 38 \mathrm{a}$ is activated via a noncanonical pathway via homodimerization. ${ }^{21}$ These data suggested that c-Abl mediates p38a activation via both canonical (Y182) and noncanonical (Y323) pathways.

To further explore the molecular mechanisms regulating the phosphorylation of p38a at $\mathrm{Y} 323$ by the kinase c-Abl, we expressed EGFP-tagged p38 $a$ and FLAG-tagged p38 $a$ in cells to examine $\mathrm{p} 38 \mathrm{a}$ homodimerization. The coexpression of $\mathrm{p} 38 \mathrm{a}$ and c-Abl dramatically increased the homodimerization of WT p38a but not Y323F mutant p38a (Figure 4f). These data indicated that p38a phosphorylation at $\mathrm{Y} 323$ by $\mathrm{c}-\mathrm{Abl}$ promotes $\mathrm{p} 38 a$ homodimerization and activates p38a via a noncanonical pathway.

C-Abl mediates p38a activation in PD model. The finding that $\mathrm{c}-\mathrm{Abl}$ phosphorylates p38a led us to investigate the role 

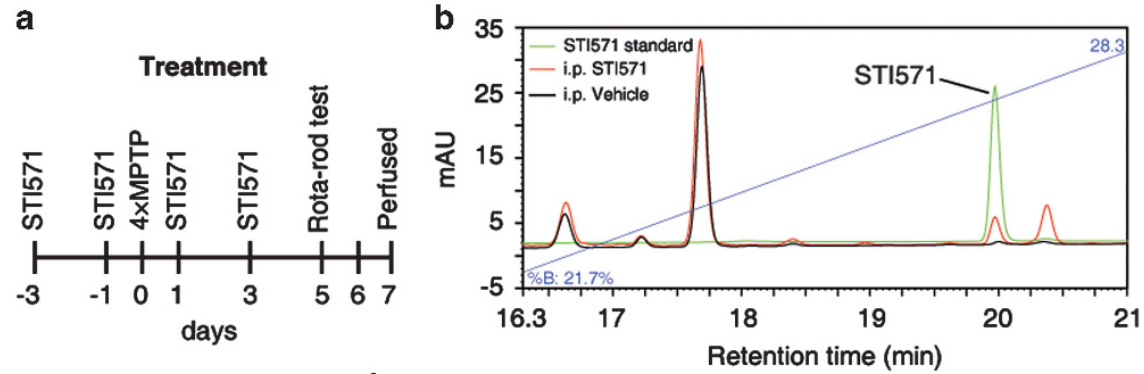

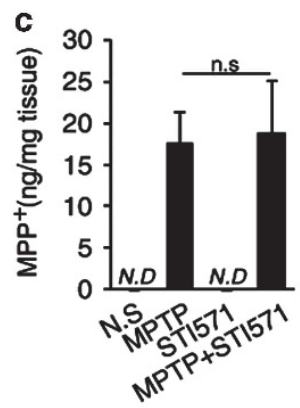

d

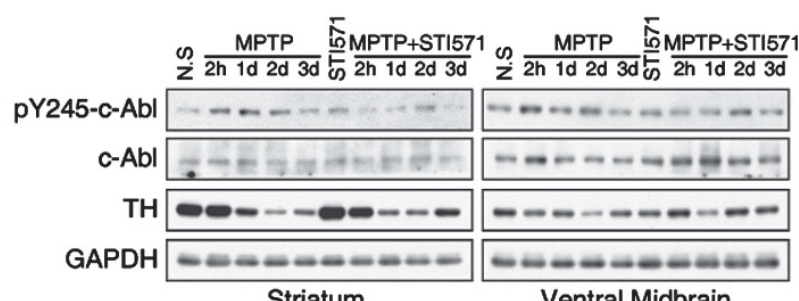

Striatum

e
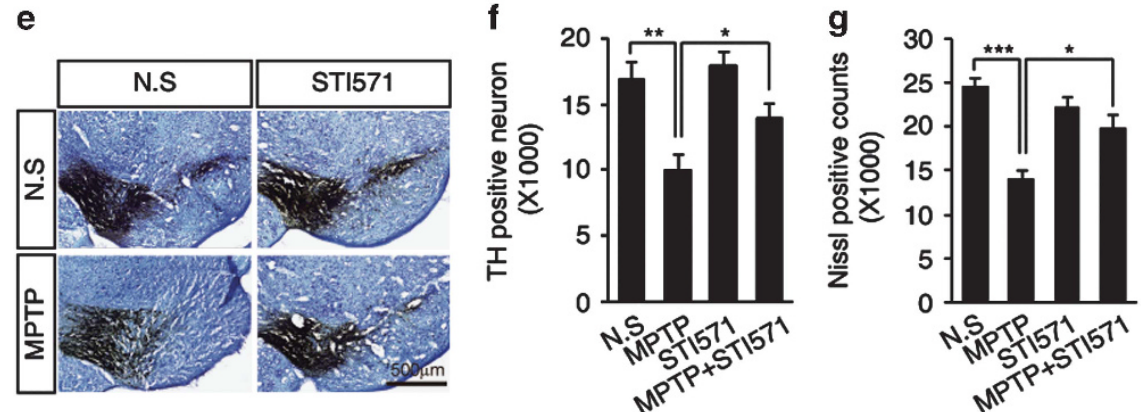

h

i

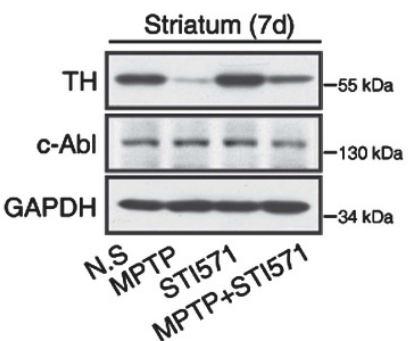

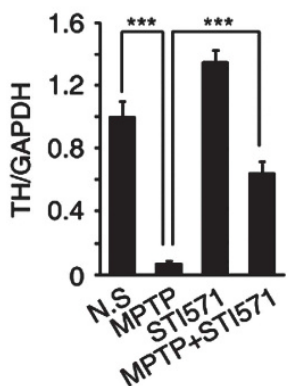

j

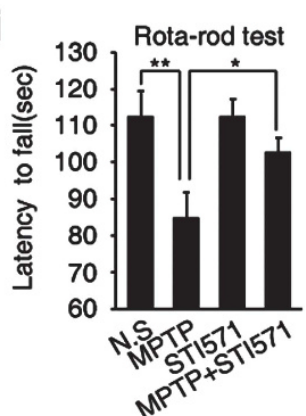

Figure 2 The c-Abl inhibitor STI571 reduces dopaminergic neuronal cell death and locomotive defects induced by acute MPTP treatment. (a) Experimental design. For drug treatment, the C57BL/6J mice were treated with STI571 (four i.p. injections of $30 \mathrm{mg} / \mathrm{kg}$ at 1-day intervals) followed by an additional injection $12 \mathrm{~h}$ after treatment with MPTP (four i.p. injections of $20 \mathrm{mg} / \mathrm{kg}$ at $2 \mathrm{~h}$ intervals). The mice were killed 7 days after the final MPTP injection. Behavior was assessed using the Rota-Rod test 5 days after the final MPTP injection. (b) Brain penetration of STI571 in ventral midbrain $2 \mathrm{~h}$ after intraperitoneal injection. (c) Mice were treated as in (a) and killed 90 min after final MPTP injection. The levels of $\mathrm{MPP}^{+}$in the striatum of the mice were measured by HPLC analysis. (d) C57BL/6J mice were treated with STI571 (i.p. injections of $30 \mathrm{mg} / \mathrm{kg}$ at 1-day intervals) and MPTP as shown in (a). Tissue was collected from the striatum and ventral midbrain at indicated time points after the final MPTP injection and was subjected to immunoblotting using an anti-pY245-c-Abl antibody to determine the level of activated c-Abl. An anti-GAPDH antibody was used as a loading control. (e) Photomicrographs of TH and Nissl co-stained sections in the substantia nigra of mice treated as in (a). (f and $\mathbf{g}$ ) The number of TH-positive neurons (f) and Nissl-stained cells $(\mathbf{g})$ as determined by stereological quantification in the substantia nigra of mice treated as in (a). Data are expressed as mean \pm S.E.M. (ANOVA, ${ }^{\star} P<0.05,{ }^{\star \star} P<0.01, n=5$ ). (h) C57BL/6J mice were treated as in (a), and tissue was collected from the striatum. The striatal tissue lysates were immunoblotted using an anti-TH antibody to examine the loss of dopaminergic neurons. (i) The normalized levels of TH. The data are expressed as mean \pm S.E.M. (ANOVA, ${ }^{* \star \star} P<0.001, n=3$ ). (j) The latency of the mice on the Rota-Rod. The data are expressed as mean \pm S.E.M. (ANOVA, ${ }^{*} P<0.05,{ }^{* *} P<0.01, n=12$ per group)

of c-Abl-p38a signaling in neurodegenerative disease models. First, treatment with 1-methyl-4-phenylpyridinium $\left(\mathrm{MPP}^{+}\right.$, the active metabolite of MPTP) increased $\mathrm{p} 38 \mathrm{a}$ phosphorylation in SH-SY5Y cells, and this phosphorylation was mitigated by c-Abl inhibition (Figure 5a). In an acute mouse model of PD, we observed that the level of p38a phosphorylated at T180/Y182 in the ventral midbrain was increased following treatment with MPTP and mitigated by 
a

a

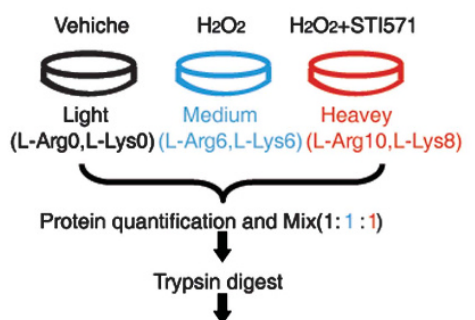

C18 purification of lysate peptides

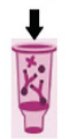

4G10 Tyrosine antibody IP

4

V

Concentration and purification of peptides

\section{1}

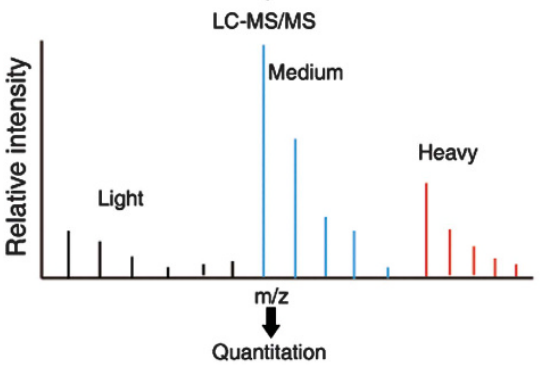

e

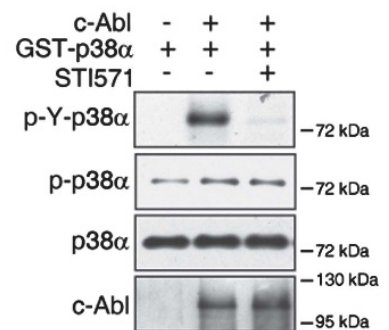

b

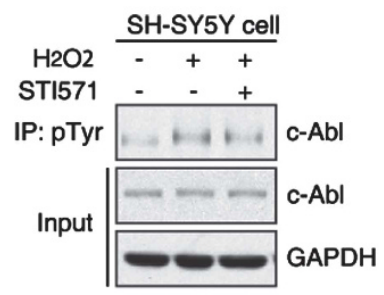

C

\begin{tabular}{l|c|c|c}
\hline Gene name & Modified Sequence & M/L & H/M \\
\hline Arg & _IMTGDTY(ph)TAHAGAK_ & 6.4986 & 0.3134 \\
\hline FAK & YMEEDSTY(ph)YK & 1.3759 & 0.6061 \\
\hline CAV1 & Y(ph)VDSEGHIY(ph)TVPIR & 1.5681 & 0.3913 \\
\hline WASP & IIY(ph)DFIEDQGGIEAVR & 1.6431 & 0.6860 \\
\hline MAPK14 & _HTDDEMT(ph)GY(ph)VATR_- & 3.6641 & 0.6978 \\
\hline MAPK1 & LADPEHDHTGFITEY(ph)VATR & 2.3203 & 0.6184 \\
\hline MAPK2 & VADPDHDHTGFIT(ph)EY(ph)VATR & 1.5472 & 0.6541 \\
\hline MAPK7 & GICTSPAEHQYFMTEY(ph)VATR & 1.9006 & 0.6227 \\
\hline
\end{tabular}

d
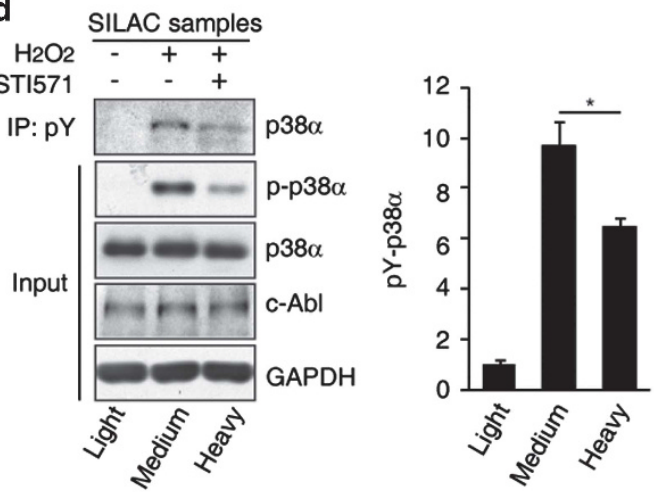

f

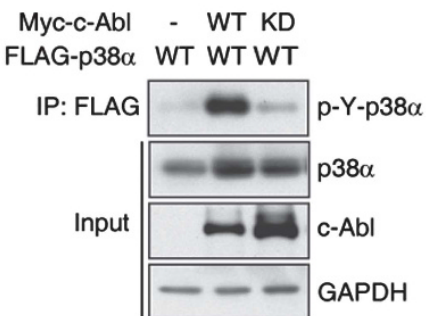

Figure 3 SILAC identifies p38 $\alpha$ as a major substrate of c-Abl during oxidative stress. (a) The experimental design in which SILAC was performed to screen for c-Abl substrates under conditions of oxidative stress. Briefly, three populations of SH-SY5Y cells were metabolically labeled with normal arginine and lysine (Arg0 and Lys0), or forms that are 6 (Arg6 and Lys6) or 10/8 Daltons heavier (Arg10 and Lys8). After treatment with $\mathrm{H}_{2} \mathrm{O}_{2}$ with or without STI571, the cell lysates were quantified and mixed together in equal portions. After digestion with trypsin, the lysates were immunoprecipitated using antibodies against phosphotyrosine. The precipitated peptides were analyzed via LC-MS. (b) The SILAC-labeled SH-SY5Y cells were untreated or treated with $800 \mu \mathrm{M} \mathrm{H}_{2} \mathrm{O}_{2}$ for 30 min in the presence or absence of $5 \mu \mathrm{M} \mathrm{STI571.} \mathrm{The} \mathrm{cell} \mathrm{lysates} \mathrm{were} \mathrm{immunoprecipitated}$ using an anti-phosphotyrosine antibody followed by immunoblotting using an antibody against c-Abl. (c) The list of identified proteins by SILAC. (d) The SH-SY5Y cell lysates used for SILAC were subjected to immunoprecipitation using an anti-phosphotyrosine antibody and immunoblotting using antibodies against p38 $\alpha$, phospho-p38 $\alpha$ (T180/Y182), c-Abl, and GAPDH. The bar graph in the right panel shows the quantification of p38 $\alpha$ tyrosine phosphorylation (ANOVA, ${ }^{*} P<0.05, n=3$ ). (e) Kinase-active c-Abl was untreated or treated with $5 \mu \mathrm{M} \mathrm{STI571}$ and then subjected to an in vitro kinase assay using full-length GST-p38 $\alpha$ as a substrate. The phosphorylation reactions were analyzed via immunoblotting using anti-phosphotyrosine and anti-phospho-p38 $\alpha$ (T180/Y182, p-p38 $\alpha$ ) antibodies. p38 $\alpha$ was tyrosine phosphorylated by c-Abl and was autophosphorylated at T180 in vitro. (f) Lysates of 293T cells transfected with FLAG-tagged p38 $\alpha$ alone or together with Myc-tagged c-Abl-WT or c-Abl-KD (kinase-dead) expression plasmids were immunoprecipitated using an anti-FLAG antibody and were analyzed via immunoblotting using an anti-phosphotyrosine antibody. p38 $\alpha$ was tyrosine phosphorylated by c-Abl kinase in vivo

treatment with STI571 (Figure 5b). Accordingly, phosphorylation of p38a in the striatum was also dramatically increased following treatment with MPTP, peaking after 3 days (Figures $5 \mathrm{c}$ and $\mathrm{d}$ ). Furthermore, we found that p38 phosphorylation sustains in the MPTP model even after c-Abl phosphorylation levels returns to the baseline, indicating that $\mathrm{c}-\mathrm{Abl}$ is an upstream kinase of p38 under oxidative stress.

To confirm that c-Abl mediates p38a phosphorylation in vivo, $\mathrm{c}-\left.\mathrm{Ab}\right|^{\text {flox/flox }}$ mice were crossed with CaMKIla-iCre transgenic mice that generate a conditional $\mathrm{KO}$ of $\mathrm{c}-\mathrm{Abl}$ in neurons. ${ }^{22}$ The age-matched WT and c-Abl KO littermates were treated with 
a

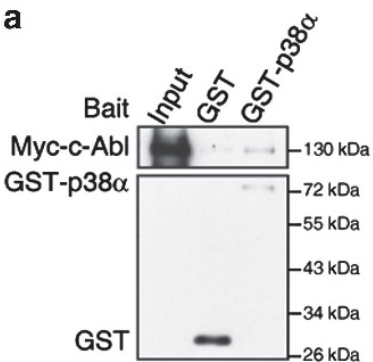

b

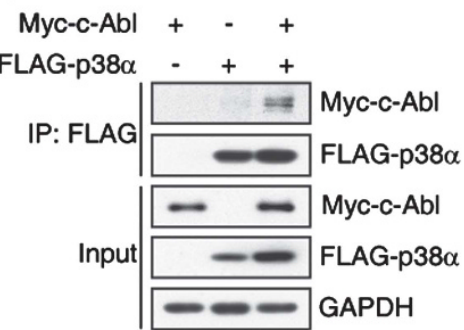

c

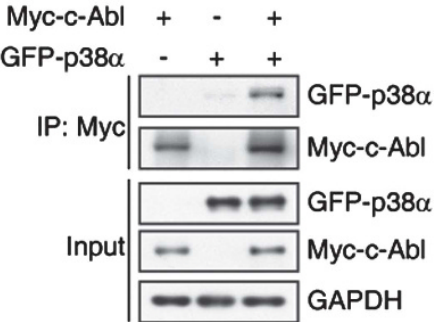

d

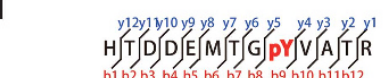

y33y32 y31y30y29y28y27y26y25y24y23y22y21 y20y19y18 y17y16y15y14y13y12y11y10 y9 y8 y7 y6 y5 y4 y3 y2 y1

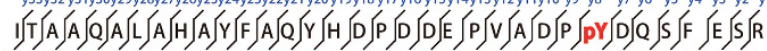

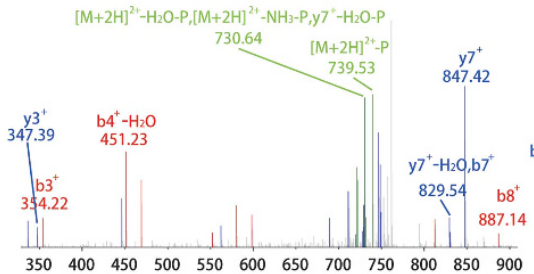

$\mathrm{m} / \mathrm{z}$

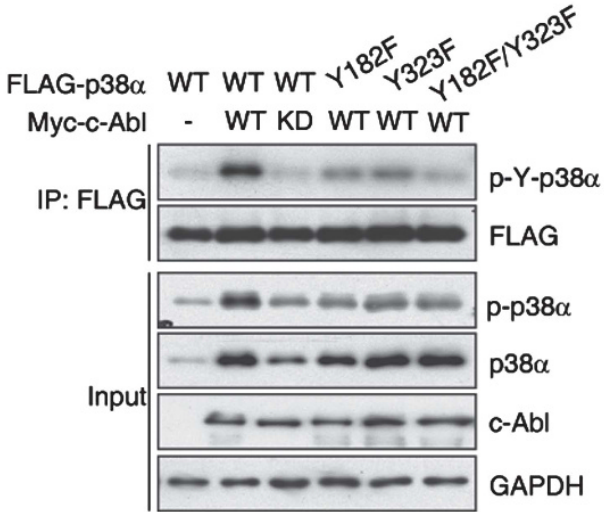

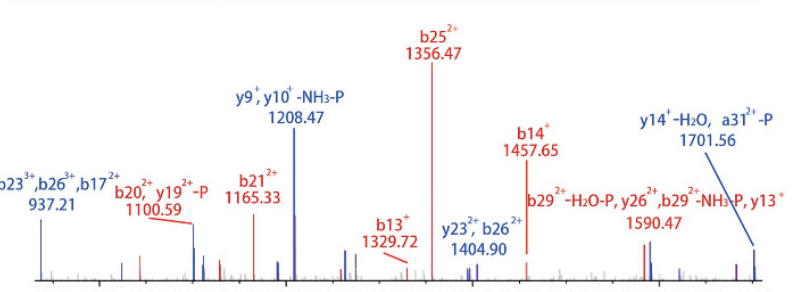

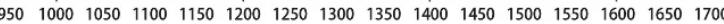

$\mathrm{m} / \mathrm{z}$

f FLAG-p38 $\alpha$ - WT WTY323F

GFP-p38 $\alpha$ WT WT WTY323F

Myc-c-Abl

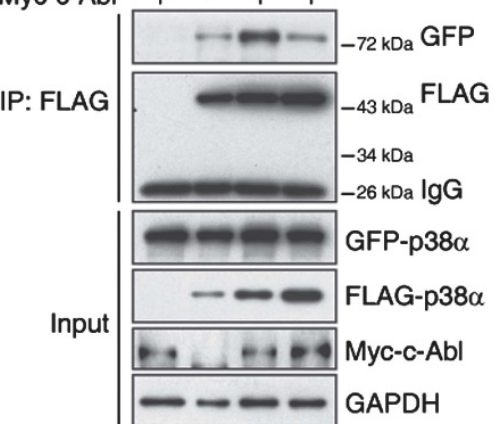

Figure 4 c-Abl interacts with p38 $\alpha$ and phosphorylates p38 $\alpha$ at $Y 182$ and $Y 323$. (a) 293T cells were transfected with Myc-tagged c-Abl and cell lysates were incubated with GST-p38 $\alpha$ or GST protein and Glutathione-Sepharose beads followed by immunoblotting with anti-Myc antibody. (b) Lysates from 293 T cells transfected with indicated plasmids were subjected to immunoprecipitation with anti-FLAG antibody, followed by immunoblotting with anti-Myc or anti-FLAG antibody. (c) Lysates from 293T cells transfected indicated plasmids were subjected to immunoprecipitation with anti-Myc antibody, followed by immunoblotting with anti-GFP or anti-Myc antibody. (d) The phosphorylation reactions from Figure 3e were subjected to SDS-PAGE followed by Coomassie Blue staining. The band corresponding to p38 $\alpha$ was excised from the gel and digested with trypsin. The phosphorylation sites were mapped via microcapillary LC-MS/MS, resulting in $77.8 \%$ coverage of the p38 $\alpha$ amino acid sequence. Two phosphopeptides consistent with p38 $\alpha$ phosphorylation at Y182 and Y323 were identified. (e) Lysates of 293T cells transfected with FLAG-tagged WT, Y182F, Y323F or Y182F, and Y323F p38 $\alpha$ alone or together with Myc-tagged c-Abl-WT or c-Abl-KD expression plasmids were immunoprecipitated using an anti-FLAG antibody and were analyzed via immunoblotting using anti-phosphotyrosine and anti-FLAG antibodies. Y182 and Y323 are sites of p38 $\alpha$ that are phosphorylated by c-Abl. (f) Lysates of 293T cells transfected with GFP-tagged WT or Y323F p38 $\alpha$ alone or with Myc-tagged c-Abl and FLAG-tagged WT or Y32F p38 $\alpha$ expression plasmids were immunoprecipitated using an anti-FLAG antibody and were analyzed via immunoblotting using anti-GFP and anti-FLAG antibodies. The c-Abl-mediated phosphorylation of p38 $\alpha$ at Y323 promotes p38 $\alpha$ dimerization

saline or MPTP (four intraperitoneal injections of $20 \mathrm{mg} / \mathrm{kg}$ at $2 \mathrm{~h}$ intervals) and killed $40 \mathrm{~h}$ after the final MPTP injection. Immunohistochemistry results show that C-Abl KO abrogated the increase in the levels of phospho-p38a in the TH-positive neurons and neuritic terminals of TH-positive neurons in the striatum (Figure $5 \mathrm{e}$ ). It has been reported that acute MPTP treatment will induce microglial activation by $\mathrm{MPP}^{+}$or agents released by injured neurons. ${ }^{23}$ Accordingly, we also found that the level of phosphorylated p38a increased in microglial cells (Figure 5e), Interestingly, less microglial activation in the striatum of neuron-specific c-Abl KO mice was observed, and this might be due to reduced damaged neurons in $\mathrm{c}-\mathrm{Abl} \mathrm{KO}$ brain (Figure 5e). Furthermore, immunoblotting showed that there was a significant decreased level of phosphorylated p38 $a$ in both ventral midbrain and striatum from c-Abl KO mice compared with WT mice upon MPTP treatment (Figure 5f). Taken together, these data suggested that c-Abl endogenously phosphorylates p38 $a$ and promotes neuronal cell death.

The p38a inhibition alleviates MPTP-induced dopaminergic neuron loss and motor defects. After establishing the role of c-Abl-p38a signaling in MPTP-induced dopaminergic 
a

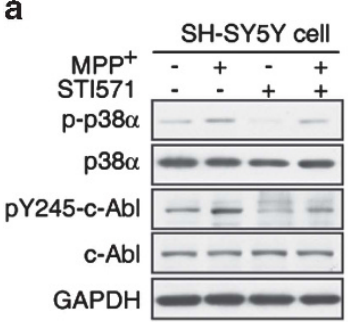

b

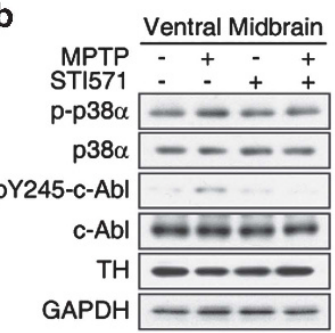
c d

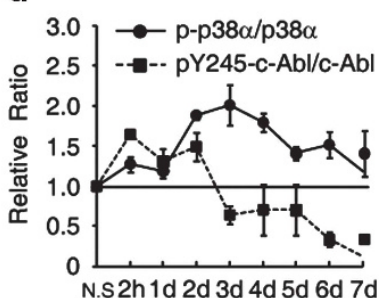

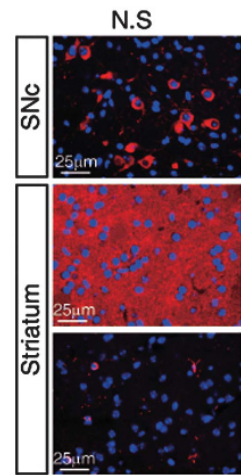

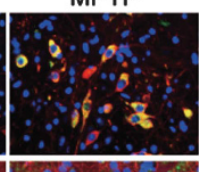

KO

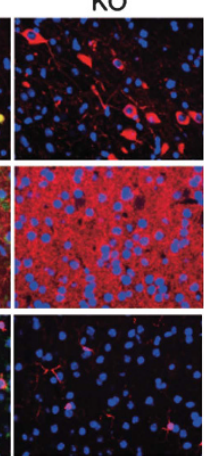

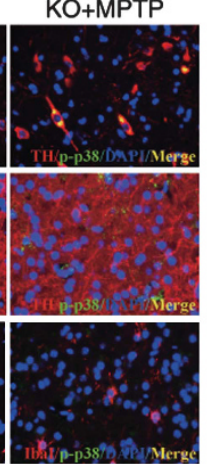

f

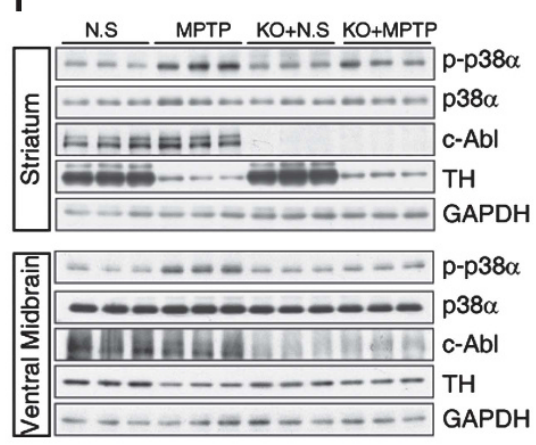

Figure 5 C-Abl mediated p38 $\alpha$ activation in the PD model. (a) SH-SY5Y cells were untreated or treated with $1 \mathrm{mM} \mathrm{MPP}^{+}$for $1 \mathrm{~h}$ in the presence or absence of $5 \mu \mathrm{M}$ STI571. The cell lysates were immunoblotted using an anti-phospho-p38 $\alpha$ (Thr180/Y182, p-p38 $\alpha$ ) antibody. (b) C57BL/6 J mice were treated with STI571 (two i.p. injections of $30 \mathrm{mg} / \mathrm{kg}$ at 1-day intervals) before MPTP treatment as in Figure 2a. Tissue was collected from ventral midbrain at $2 \mathrm{~h}$ after the final MPTP injection and subjected to immunoblotting with anti-phospho-p38 $\alpha$ (Thr180/Y182, p-p38 $\alpha$ ) antibody or anti-pY245-c-Abl antibody. An anti-GAPDH antibody was used as a loading control. (c) C57BL/6J mice were treated with saline or MPTP (four i.p. injections of $20 \mathrm{mg} / \mathrm{kg}$ at $2 \mathrm{~h}$ intervals), and striatal tissue was collected at $2 \mathrm{~h}$ and on each of 7 consecutive days after MPTP treatment. The striatal tissue lysates were immunoblotted using an anti-phospho-p38 $\alpha(\operatorname{Thr} 180 / Y 182, \mathrm{p}-\mathrm{p} 38 \alpha)$ antibody to detect the level of activated p38 $\alpha$, using antiphospho-Y245-c-Abl (pY245-c-Abl) to detect tyrosine-phosphorylated c-Abl, using an anti-actin antibody as a loading control, and using a TH antibody to detect the number of surviving dopaminergic neurons after MPTP treatment. (d) The normalized levels of p-p38 $\alpha$ and pY245-c-Abl. The data are expressed as mean \pm S.E.M. (e) Immunofluorescence images of TH/lbal (red), phospho-p38 $\alpha$ (green), and merged staining (yellow) in substantia nigra or striatum from MPTP-treated c-Abl KO mice or WT littermates. (f) Lysates of striatum or ventral midbrain from WT and c-Abl KO littermate mice after treatment with saline or MPTP were immunoblotted with anti-phospho-p38 $\alpha$ (Thr180/Y182, p-p38 $\alpha$ ) antibody

neuronal death, we further examined whether p38a inhibition might provide therapeutic value for oxidative stress-induced PD mice. C57BL/6J mice were treated with either saline or MPTP (four intraperitoneal injections of $20 \mathrm{mg} / \mathrm{kg}$ at $2 \mathrm{~h}$ intervals) with or without the p38a-specific inhibitor SB203580 (Figure 6a). We first confirmed brain penetration of intraperitoneally injected SB203580 in the ventral midbrain by high-performance liquid chromatography (HPLC) analysis (Figure 6b), and we also found that intraperitoneal injection of SB203580 did not affect the concentration of $\mathrm{MPP}^{+}$in the striatum (Figure $6 \mathrm{c}$ ). Interestingly, SB20580 treatment abolished MPTP-induced p38a phosphorylation in both TH-positive neurons from substantia nigra and neuritic terminals of TH-positive neurons in the striatum (Figure $6 \mathrm{~d}$ ). Moreover, the inhibition of p38a markedly prevented the MPTP-induced loss of TH-positive neurons or Nissl-stained cells in the substantia nigra (Figures 6e-g). In addition, MPTP-induced downregulation of $\mathrm{TH}$ expression in the striatum could be rescued by p38a inhibitor (Figures $6 \mathrm{~h}$ and i). Rota-Rod assays showed that SB203580 treatment significantly improved the motor activity of the MPTP-treated PD mice (Figure 6j). Taken together, these data indicated that p38 $a$ inhibition may represent a strategy for treating oxidative stressinduced PD.

\section{Discussion}

The major finding of this study is that $c-A b l$ mediates the activation of p38a in mice with MPTP-induced PD. First, c-Abl was activated following treatment with MPTP. The conditional $\mathrm{KO}$ of $\mathrm{c}-\mathrm{Abl}$ in neurons or treatment with a $\mathrm{c}-\mathrm{Abl}$ inhibitor rescued the MPTP-induced loss of dopaminergic neurons. Second, based on SILAC analysis, we identified p38a as a novel direct target of c-Abl. We also confirmed that c-Abl phosphorylates p38 $a$ and activates p38 $a$ by increasing p38 $a$ dimerization. Finally, we showed that inhibiting p38a rescues dopaminergic neurons from MPTP-induced death, suggesting that treatment with an inhibitor of c-Abl or p38a may serve as an effective therapy for PD.

The nonreceptor tyrosine kinase c-Abl is activated by cellular stress ${ }^{24}$ and plays a critical role in chronic myeloid leukemia that has been commonly treated with the c-Abl inhibitor STI571. ${ }^{25}$ Recently, extensive studies have been performed on c-Abl in the nervous system to investigate its role in neurodegenerative disease..$^{9-14,26}$ For example, c-Abl kinase has been reported to regulate the accumulation of AIMP2 (aminoacyl tRNA synthetase complex-interacting multifunctional protein 2), FBP1 (far upstream elementbinding protein 1), and $a$-synuclein in PD models. ${ }^{9,10,11}$ Here, by using the SILAC analysis, we identified p38a as a major 
a

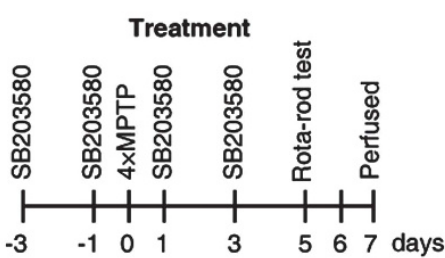

b

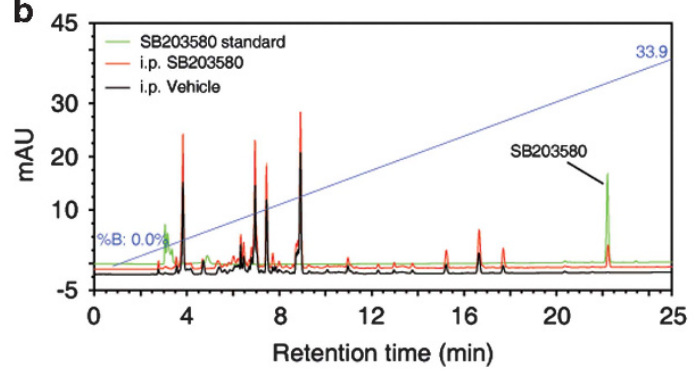

c

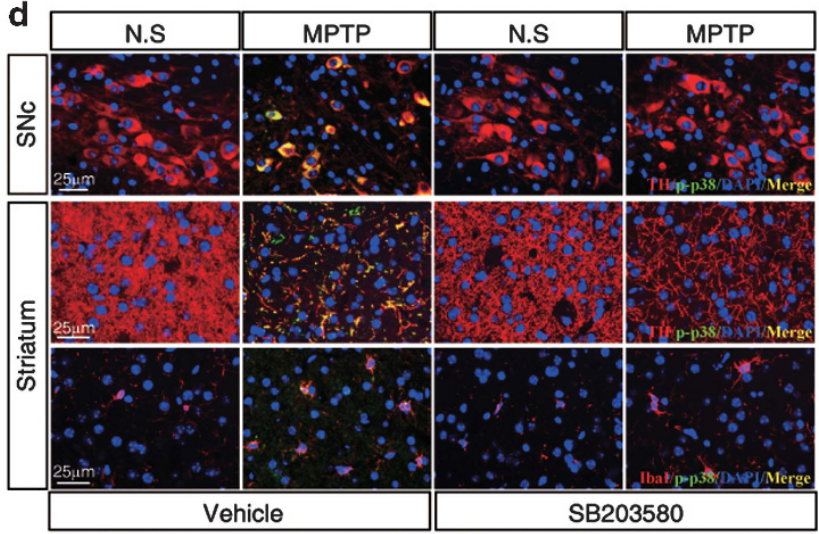

e

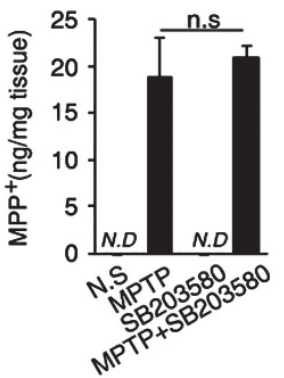

f
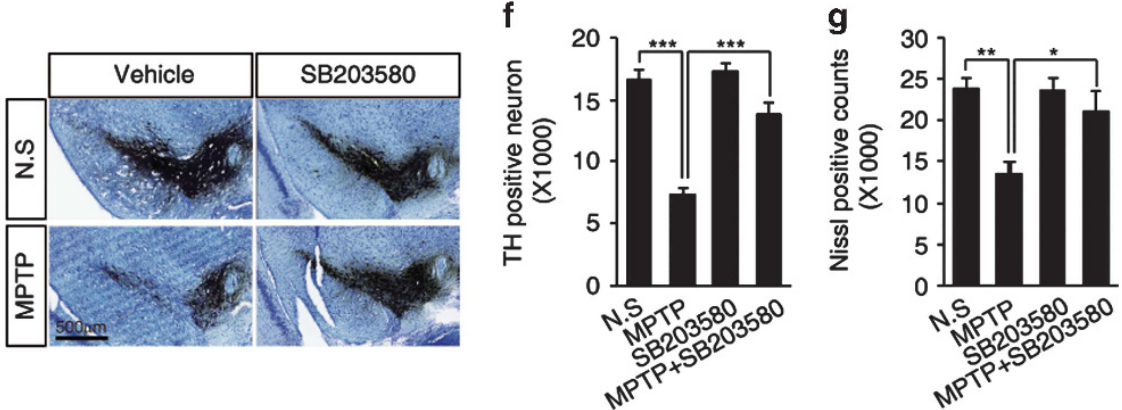

h

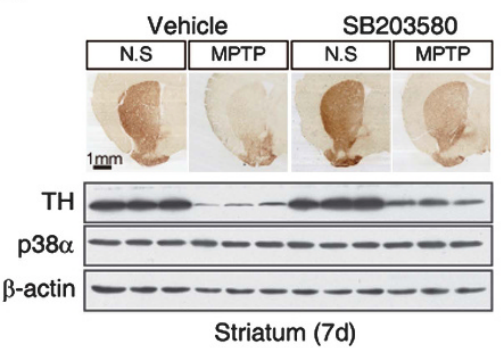

i

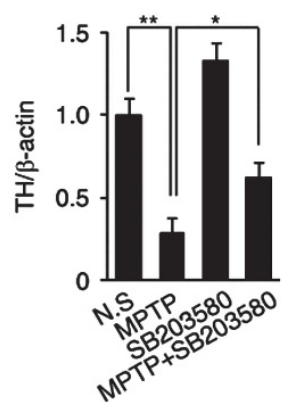

j

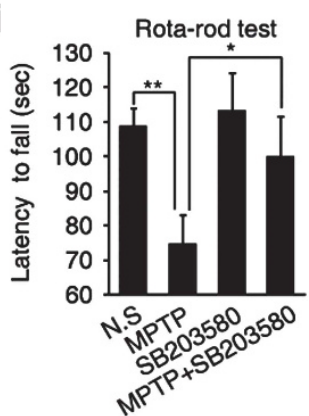

Figure 6 The p38 $\alpha$ inhibitor SB203580 protects against MPTP-induced dopaminergic neuronal death and motor deficits. (a) Experimental design. C57BL/6J mice were treated with SB203580 (four i.p. injections of $5 \mathrm{mg} / \mathrm{kg}$ at 1-day intervals) followed by an additional injection $12 \mathrm{~h}$ after treatment with MPTP (four i.p. injections of $20 \mathrm{mg} / \mathrm{kg}$ at $2 \mathrm{~h}$ intervals). The mice were killed 7 days after the final MPTP injection. For behavioral testing, the mice were trained on the Rota-Rod at 10 r.p.m. for 2 days before any drug treatment. At 5 days after the final MPTP injection, behavior was tested using the Rota-Rod. (b) Brain penetration of SB203580 was detected by HPLC analysis in ventral midbrain after $2 \mathrm{~h}$ of intraperitoneal injection. (c) Mice were treated as in (a) and killed $90 \mathrm{~min}$ after final MPTP injection. The levels of MPP ${ }^{+}$in the striatum were measured. ND, not detected ( $n=3$ per group). (d) Immunofluorescence images of TH/lbal (red), phospho-p38 $\alpha$ (green), and merged staining (yellow) in substantia nigra or striatum from the MPTP alone or MPTP/SB203580-treated mice. (e) Photomicrographs of TH and Nissl co-stained sections in the substantia nigra of mice treated as in (a). (f and $\mathbf{g})$ The number of TH-positive neurons (f) and Nissl-stained cells $(\mathbf{g})$ was determined by stereological quantification for $(\mathbf{e})$. The data are presented as mean \pm S.E.M. (ANOVA, ${ }^{*} P<0.05,{ }^{* \star *} P<0.001, n=5$ ). (h) Photomicrographs of TH-immunostained sections of the striatum from the mice treated as in (a) (upper panel). The striatal tissue lysates were immunoblotted with anti-TH antibody (bottom panel). (i) Normalized levels of TH protein. The data are expressed as mean \pm S.E.M. (ANOVA, ${ }^{*} P<0.05,{ }^{* *} P<0.01, n=3$ ). (j) The latency of the mice on the Rota-Rod. The data are expressed as mean \pm S.E.M. (ANOVA, ${ }^{*} P<0.05,{ }^{* \star} P<0.01, n=11-15$ per group) 
substrate of c-Abl in the process of oxidative stress-induced neuronal cell death.

We previously showed that c-Abl phosphorylates mammalian Ste20-like kinase 1 (MST1) and enhances MST1-mediated signaling to promote oxidative stressinduced neuronal death. ${ }^{6}$ The kinase MST1 may act upstream of p38a and c-Jun N-terminal kinase (JNK) in response to oxidative stress. ${ }^{27,28}$ In this study, we clearly demonstrated that c-Abl directly phosphorylates p38 $a$ and plays a critical role in the MPTP-induced pathogenesis of PD. However, the role of MST1 in c-Abl-mediated p38a activation requires further investigation.

The p38a is a subgroup of MAPKs that mediate responses to extracellular stimulation. ${ }^{29}$ The $\mathrm{p} 38 a$ is typically activated by an upstream MAPK kinase such as MKK3 or MKK6. ${ }^{30}$ The $T$ cell antigen receptor signaling pathway bypasses the typical MAPK cascade and activates p38 $a$ via phosphorylation at Tyr-323 followed by autophosphorylation of p38a in the activation loop. ${ }^{31}$ We demonstrated that $\mathrm{c}-\mathrm{Abl}$ directly phosphorylates and activates p38a in response to oxidative stress to induce neuronal death. Furthermore, we showed that a p38a inhibitor rescued dopaminergic neurons from MPTP-induced death; thus, p38a may represent a therapeutic target for PD treatment.

Various inhibitors including STI571 and SB203580 have been used in the treatment of diseases outside of central nervous system because of their low brain penetration. Moreover, the therapeutic limitation of these inhibitors is caused by their specificity. Therefore, the development of high brain-permeable and target-specific inhibitors of c-Abl and p38 would facilitate the therapeutic treatment for the neurodegenerative diseases.

In summary, the present study identified p38a as a novel substrate of c-Abl during MPTP-induced death of dopaminergic neurons, providing further support for the crucial role of c-Abl in the development of PD. In future, it would be valuable to explore the efficacy of c-Abl-p38a inhibition as a therapeutic strategy for PD.

\section{Materials and Methods \\ Animals. Mice were maintained under conditions of a 12-h light/dark cycle at $23^{\circ} \mathrm{C}$ and were provided with food and water ad libitum in the Animal Care Facility at the Institute of Biophysics (Beijing, China). All experiments involving animals were approved by and conformed to the guidelines of the institutional animal care and use committee at the Institute of Biophysics of the Chinese Academy of Sciences (Beijing, China).}

Generation of mice with a conditional c-Abl KO in neurons.

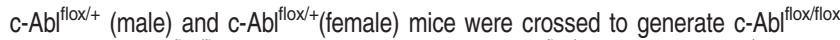

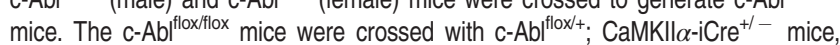
and the offspring c-Abl floxflox; CaMKIl $\alpha-\mathrm{ICre}^{++}$mice (c-Abl KO) and c-Ablloxfflox mice (WT) were used in the experiments. This approach enabled Cre recombinase to inactivate the c-Abl gene specifically in cells in which the CaMKIl $\alpha$ promoter is active. The floxed $c-A b l$ gene was identified via PCR using primer-1 (5'-CAGCAACCGGCTTGCATG-3') and primer-2 (5'- AGGCCTTCTTCCTGATAG TC-3'), yielding PCR products of 200 and $230 \mathrm{bp}$ for the WT and floxed alleles, respectively. For PCR of the CaMKIll $\alpha$-Cre allele, we used the forward primer $5^{\prime}$-GGTT CTCCGTTTGCACTCAGGA-3' and the reverse primer $5^{\prime}$-CCTGTTGTTCAG CTTGCACCAG-3', yielding a 350-bp product.

Drug treatment in vivo. Adult C57BL/6J mice were administered four

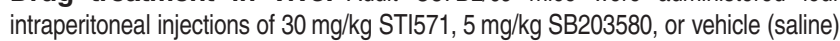

at 1-day intervals at $12 \mathrm{~h}$ before and after MPTP injection (Figures 2a and 6a). The mice were administered four intraperitoneal injections of $20 \mathrm{mg} / \mathrm{kg}$ MPTP as previously described. ${ }^{32}$ At 7 days after the final MPTP injection, the animals were killed, and the striatum was dissected and processed for western blot analysis. In some cases, the animals were perfused with $4 \%$ paraformaldehyde in $0.1 \mathrm{M}$ phosphate buffer ( $\mathrm{pH} 7.4)$, and $5 \mu \mathrm{m}$ coronal paraffinized sections were prepared for immunohistochemistry assays.

TSA immunohistochemistry. To detect the phosphorylation of p38, tyramide signal amplification (TSA) method was performed according to the protocol from the manufacturer (PerkinElmer, Waltham, MA, USA). Coronal sections were incubated with anti-phospho-p38 (1:100, Cell Signaling Technology, 9216, Beverly, MA, USA) and followed by horseradish peroxidase (HRP) reaction and visualization with TSA kit. The sections were then incubated with rabbit polyclonal anti-TH (1:200, Pel Freez Biologicals, P40101, Rogers, AR, USA) or anti-lbal (1:100, Wako, 019-19741, Chuo-Ku, Osaka, Japan) and visualized by immunofluorescent microscopy.

Measurement of striatal MPP ${ }^{+}$levels. Mice were killed 90 min after the final MPTP injection, and striata were dissected and sonicated. After centrifugation, the supernatant was added 4 volume acetonitrile to precipitated protein. The supernatants were dehydrated and resolved in $100 \mu \mathrm{l}$ water. Then, $50 \mu \mathrm{l}$ of supernatant was injected into the Ultimate XB-C18 column $(4.6 \times 250 \mathrm{~mm}, 5 \mu \mathrm{m}$, Welch, Shanghai, China) and eluted with ultrapure water (1\%o trifluoroacetate)/ acetonitrile ( $1 \%$ o trifluoroacetate) in a gradient manner. Finally, the $\mathrm{MPP}^{+}$was detected at $280 \mathrm{~nm}$.

Brain permeability analysis of STI571 and SB203580. Mice were intraperitoneally injected with STI571 $(150 \mathrm{mg} / \mathrm{kg})$ or SB203580 $(50 \mathrm{mg} / \mathrm{kg})$. After $2 \mathrm{~h}$, the ventral midbrain was collected and sonicated followed by centrifugation at 14000 r.p.m. for $15 \mathrm{~min}$. Next, 4 volume acetonitrile was added into the supernatant to precipitated proteins and followed by centrifugation at 14000 r.p.m. for 15 min. The second supernatant was dehydrated and resolved in $100 \mu \mathrm{l}$ water. Then, $50 \mu \mathrm{l}$ of supernatant was injected onto a Ultimate XB-C18 column $(4.6 \times 250 \mathrm{~mm}, 5 \mu \mathrm{m}$, Welch) and eluted with ultrapure water (1\%o trifluoroacetate)/acetonitrile (1\%o trifluoroacetate) in a gradient manner. Finally, STI571 was detected at $275 \mathrm{~nm}$ and SB203580 was detected at $300 \mathrm{~nm}$.

Stereological analysis. The brains were post-fixed using $4 \%$ paraformaldehyde, cryoprotected in 30\% sucrose, and processed for immunohistochemistry. Coronal sections $40 \mu \mathrm{m}$ in thickness were sliced throughout the brain, including the substantia nigra and striatum, and every fourth section was analyzed. For TH labeling, the slices were treated with a 1:1000 dilution of rabbit polyclonal anti-TH (P40101, Pel Freez Biologicals) followed by biotinylated goat anti-rabbit IgG and streptavidin-conjugated HRP (Vectastain ABC kit, Vector Laboratories, Burlingame, CA, USA). Positive immunostaining was visualized using $3,3^{\prime}$-diaminobenzidine (DAB) followed by a reaction with hydrogen peroxide (DAB kit, Vector Laboratories). Stained sections were mounted onto slides and counterstained with Nissl (1\% Toluidine Blue). The total numbers of TH-stained or Nissl-stained neurons from the substantia nigra pars compacta region were counted using the Optical Fractionator tool in Stereo Investigator software (MicroBrightfield, Williston, VT, USA).

Motor coordination test. Motor performance was estimated using an accelerating Rota-Rod (Panlab, LE8200, Energia, Cornella, Spain). After mice were placed on the rod, the timer was started. The mice were trained on the Rota-Rod at 10 r.p.m. three times per day (at $1 \mathrm{~h}$ intervals) for 2 days before testing. During testing, the rod accelerated from 4 to 40 r.p.m. over a period of $300 \mathrm{~s}$. The mice remaining on the apparatus after $600 \mathrm{~s}$ were removed, and the time was recorded as $600 \mathrm{~s}$. Each result represents the average endurance of three consecutive measurements performed at $1 \mathrm{~h}$ intervals.

Plasmids and transfection. The plasmids used were pCMV-Myc-c-Abl WT and KD as previously described. ${ }^{6}$ The $3 \times$ FLAG-tagged p38 $\alpha$ constructs inserted into the PCMV10-3XFLAG expression vector were created using the mouse CDNA library. The $\mathrm{Y} 182 \mathrm{~F}$ and $\mathrm{Y} 323 \mathrm{~F}$ mutants of $\mathrm{p} 38 \alpha$ were generated via site-directed mutagenesis. All mutations were verified via sequencing. Fragments of the GSTp38 $\alpha$ plasmids were cloned into pGEX6P1 at the BamHI and Nott restriction sites via PCR. Unless stated otherwise, all transfections were performed in complete 
medium containing Vigofect (Vigorous Biotechnology, Beijing, China) according to the manufacturer's instructions.

SILAC labeling. 'Light', 'medium', and 'heavy' arginine (Arg0 ${ }^{12} \mathrm{C}_{6}^{14} \mathrm{~N}_{4}$, Arg6 ${ }^{13} \mathrm{C}_{6}^{14} \mathrm{~N}_{4}$, and $\operatorname{Arg} 10{ }^{13} \mathrm{C}_{6}^{15} \mathrm{~N}_{4}$, respectively) and lysine (Lys $0{ }^{12} \mathrm{C}_{6}^{14} \mathrm{~N}_{2}$, Lys6 ${ }^{13} \mathrm{C}_{6}^{14} \mathrm{~N}_{2}$, and Lys ${ }^{13} \mathrm{C}_{6}^{15} \mathrm{~N}_{2}$, respectively) and DMEM deficient in arginine and lysine were purchased from Pierce (Waltham, MA, USA). Dialyzed FBS and penicillin/ streptomycin were purchased from Gibco (Waltham, MA, USA). SILAC media were prepared using 10\% dialyzed FBS, $1 \%$ penicillin/streptomycin, and $50 \mathrm{mg} / \mathrm{l}$ arginine and lysine. The cells were cultured for a minimum of eight passages. The labeling efficiency was determined via LC-MS. The labeling efficiency was 100\%, and $<5 \%$ proline conversion was observed.

Identification of tyrosine-phosphorylated peptides. Labeled SH-SY5Y cells were seeded at $2.0 \times 10^{6}$ cells per $15 \mathrm{~cm}$ dish. When the cells reached $85 \%$ confluence, they were left untreated or were treated with $800 \mu \mathrm{M}$ $\mathrm{H}_{2} \mathrm{O}_{2}$ for $30 \mathrm{~min}$. In some groups, this treatment was preceded by a 1-h pretreatment with $5 \mu \mathrm{M}$ STI571. The cells were harvested in $9 \mathrm{M}$ urea sample buffer (9 M urea, $20 \mathrm{mM}$ HEPES, pH 8, $1 \mathrm{mM} \mathrm{Na}_{3} \mathrm{VO}_{4}, 2.5 \mathrm{mM} \mathrm{Na}_{4} \mathrm{P}_{2} \mathrm{O}_{7}$, and $1 \mathrm{mM}$ $\beta$-glycerophosphate). Then, $10 \mathrm{mg}$ of protein from each group of labeled cells was mixed. The mixed cell lysates were sonicated, centrifuged, reduced, alkylated, and digested with trypsin overnight at room temperature. To ensure complete digestion before purification, the cell lysates were analyzed via SDS-PAGE electrophoresis followed by Coomassie Blue staining. The digested lysates were acidified in $1 \%$ trifluoroacetate (TFA), and the peptides were purified using C18 Sep-Pak columns (WAT051910; Waters, Milford, MA, USA). The columns were washed with 8 volumes of $0.1 \%$ TFA. Next, the peptides were eluted with $40 \%$ acetonitrile $10.1 \%$ TFA and then dried. The tyrosine-phosphorylated peptides were isolated using $40 \mu \mathrm{l}$ of an agarose-conjugated phosphotyrosine antibody (4G10; Millipore, Billerica, MA, USA) in immunoprecipitation (IP) buffer (50 mM MOPS/NaOH, pH 7.2, $10 \mathrm{mM} \mathrm{Na} 2 \mathrm{HPO}_{4}$, and $50 \mathrm{mM} \mathrm{NaCl}$ ). The immunoprecipitates were washed twice with $1 \mathrm{ml}$ of IP buffer and then three times with $1 \mathrm{ml}$ of water. The tyrosine-phosphorylated peptides were eluted in $100 \mu \mathrm{l}$ of $0.15 \%$ TFA at room temperature. These peptides were concentrated and purified using ZipTip Pipette Tips (ZTC18M008; Millipore) according to the manufacturer's instructions. The concentrated and purified peptides were analyzed via LC-MS using an LTQ Orbitrap XL (Thermo Scientific, Waltham, MA, USA).

Co-immunoprecipitation and immunoblotting. Cells for co-immunoprecipitation were lysed in buffer containing $50 \mathrm{mM}$ HEPES, pH 7.9, $150 \mathrm{mM} \mathrm{NaCl}$, $10 \%$ Glycerol, $1 \%$ Triton- $100,1.5 \mathrm{mM} \mathrm{MgCl}, 0.1 \mathrm{M} \mathrm{NaF}, 1 \mathrm{mM}$ ethylene glycol tetraacetic acid (EGTA), $2 \mathrm{mM}$ phenylmethylsulfonyl fluoride, $2 \mu \mathrm{g} / \mathrm{ml}$ Aprotinin and Leupeptin, and $1 \mathrm{mM}$ sodium vanadate. Lysates were centrifuged at $12000 \times \mathrm{g}$ for 15 min at $4{ }^{\circ} \mathrm{C}$ before immunoprecipitation and precleared with $2 \mu \mathrm{l} \mathrm{lgG}$ and protein $\mathrm{G}$ agarose beads at $4^{\circ} \mathrm{C}$ for $2 \mathrm{~h}$. Following the removal of the beads by centrifugation, lysates were incubated with appropriate antibodies in the presence of $30 \mu \mathrm{l}$ of protein $\mathrm{G}$ agarose beads for at least $2 \mathrm{~h}$ at $4^{\circ} \mathrm{C}$. Tissues or cells for immunoblotting were lysed in buffer containing $50 \mathrm{mM}$ HEPES, pH 7.4, $150 \mathrm{mM}$ $\mathrm{NaCl}, 1 \%$ Nonidet P-40, 0.1\% deoxycholate, $0.05 \%$ SDS, $0.1 \mathrm{M} \mathrm{NaF}, 1 \mathrm{mM}$ EGTA, $2 \mathrm{mM}$ phenylmethylsulfonyl fluoride, $2 \mu \mathrm{g} / \mathrm{ml}$ aprotinin and leupeptin, and $1 \mathrm{mM}$ sodium vanadate. Protein concentration was determined using a Bio-Rad protein assay kit (Bio-Rad, Hercules, CA, USA). Proteins were separated on a $10 \%$ polyacrylamide gel and transferred to a methanol-activated PVDF membrane (GE Healthcare, Little Chalfont, Buckinghamshire, UK). The membrane was blocked for $1 \mathrm{~h}$ in Tris-buffered saline and Tween-20 (TBST) containing 5\% milk and subsequently probed with primary antibodies overnight at $4^{\circ} \mathrm{C}$. After incubating for $1 \mathrm{~h}$ with goat-anti-mouse or goat-anti-rabbit HRP-conjugated secondary antibodies (GE Healthcare), protein level was detected with Super Signal West Pico and Femto Luminol reagents (Thermo Scientific). The antibodies used were anti-c-Abl (2862, Cell Signaling Technology, Cambridge, MA, USA), anti-phosphoc-Abl (2861, Cell Signaling Technology), anti-p38 (9212, Cell Signaling Technology), anti-phospho-p38 (9211, Cell Signaling Technology), anti-TH (2792, Cell Signaling Technology), anti-phospho-tyrosine (4G10, Millipore), anti-Myc (MBL, Woburn, MA, USA), anti-FLAG (Sigma, St. Louis, MO, USA), anti-GFP (Invitrogen, Waltham, MA, USA), anti- $\beta$-actin (60008-1-lg, Proteintech Group, Campbell Park, Chicago, IL, USA), and anti-GAPDH (CW0266A, CWBiotech, Beijing, China).

In vitro kinase assay. Recombinant active c-Abl kinase (Millipore, Billerica, MA, USA) was incubated in $20 \mathrm{mM}$ Tris, $\mathrm{pH} 7.5,10 \mathrm{mM} \mathrm{MgCl}, 100 \mu \mathrm{M}$ ATP, and
$1 \mu \mathrm{g}$ of the substrate GST-p38 $\alpha$. After incubation at room temperature for $30 \mathrm{~min}$, the kinase reaction products were separated via SDS-PAGE and analyzed via immunoblotting using the indicated antibodies.

Statistical analysis. The intensity of the western blot bands was determined using ImageJ software (NIH, Bethesda, MD, USA). Statistical analyses were performed via one-way analysis of variance (ANOVA) followed by Tukey's post hoc test or via a two-tailed Student's $t$-test. The data are presented as mean \pm S.E.M. ${ }^{*} P<0.05,{ }^{* *} P<0.01$, and ${ }^{* * *} P<0.001$ denote the significance thresholds.

\section{Conflict of Interest}

The authors declare no conflict of interest.

Acknowledgements. We thank Dr. Yong Cang for the c-Abl floxflox mice and Dr. Xiang Yu for the CamKIll $\alpha$-iCre mice. We thank Dr. Peng Xue and Dr. Fuquan Yang for the mass-spec technical help. We thank Dr. Lili Niu for the HPLC technical help. We also thank the members of the Yuan laboratory for critical reading of the manuscript and helpful discussion. This work was supported by the National Science Foundation of China (Grant Nos. 81125010 and 81030025), the National Basic Research Program of China (973-2011CB504105 to ML, 973-2012CB910701 and 2013DFA31990 to ZY), and Cross-disciplinary Collaborative Teams Program for Science, Technology and Innovation (2014-2016) from Chinese Academy of Sciences.

1. Savitt JM, Dawson VL, Dawson TM. Diagnosis and treatment of Parkinson disease: molecules to medicine. J Clin Invest 2006; 116: 1744-1754.

2. Gasser T. Molecular pathogenesis of Parkinson disease: insights from genetic studies. Exp Rev Mol Med 2009; 11: e22.

3. Dauer W, Przedborski S. Parkinson's disease: mechanisms and models. Neuron 2003; 39: 889-909.

4. Langston JW, Ballard P, Tetrud JW, Irwin I. Chronic Parkinsonism in humans due to a product of meperidine-analog synthesis. Science 1983; 219: 979-980.

5. Sirvent A, Benistant C, Roche S. Cytoplasmic signalling by the c-Abl tyrosine kinase in normal and cancer cells. Biol Cell 2008; 100: 617-631.

6. Xiao L, Chen D, Hu P, Wu J, Liu W, Zhao Y et al. The c-Abl-MST1 signaling pathway mediates oxidative stress-induced neuronal cell death. J Neurosci 2011; 31: 9611-9619.

7. Liu W, Wu J, Xiao L, Bai Y, Qu A, Zheng Z et al. Regulation of neuronal cell death by c-AblHippo/MST2 signaling pathway. PLoS One 2012; 7: e36562.

8. Cancino GI, Perez de Arce K, Castro PU, Toledo EM, von Bernhardi R, Alvarez AR. c-Abl tyrosine kinase modulates tau pathology and $\mathrm{Cdk5}$ phosphorylation in $\mathrm{AD}$ transgenic mice. Neurobiol Aging 2011; 32: 1249-1261.

9. Ko HS, Lee Y, Shin JH, Karuppagounder SS, Gadad BS, Koleske AJ et al. Phosphorylation by the $\mathrm{c}-\mathrm{Abl}$ protein tyrosine kinase inhibits parkin's ubiquitination and protective function. Proc Natl Acad Sci USA 2010; 107: 16691-16696.

10. Imam SZ, Zhou Q, Yamamoto A, Valente AJ, Ali SF, Bains M et al. Novel regulation of parkin function through c-Abl-mediated tyrosine phosphorylation: implications for Parkinson's disease. J Neurosci 2011; 31: 157-163.

11. Mahul-Mellier AL, Fauvet B, Gysbers A, Dikiy I, Oueslati A, Georgeon S et al. $\mathrm{c}$-Abl phosphorylates alpha-synuclein and regulates its degradation: implication for alphasynuclein clearance and contribution to the pathogenesis of Parkinson's disease. Hum Mol Genet 2014: 23: 2858-2879.

12. Hebron ML, Lonskaya I, Moussa CE. Nilotinib reverses loss of dopamine neurons and improves motor behavior via autophagic degradation of alpha-synuclein in Parkinson's disease models. Hum Mol Genet 2013; 22: 3315-3328.

13. Imam SZ, Trickler W, Kimura S, Binienda ZK, Paule MG, Slikker W Jr et al. Neuroprotective efficacy of a new brain-penetrating C-Abl inhibitor in a murine Parkinson's disease model. PLoS One 2013; 8: e65129.

14. Karuppagounder SS, Brahmachari S, Lee Y, Dawson VL, Dawson TM, Ko HS. The c-Abl inhibitor, nilotinib, protects dopaminergic neurons in a preclinical animal model of Parkinson's disease. Sci Rep 2014; 4: 4874

15. Dai H, Marbach P, Lemaire M, Hayes M, Elmquist WF. Distribution of STI-571 to the brain is limited by P-glycoprotein-mediated efflux. J Pharmacol Exp Ther 2003; 304: 1085-1092.

16. Mahul-Mellier AL, Fauvet B, Gysbers A, Dikiy I, Oueslati A, Georgeon S et al. c-Abl phosphorylates alpha-syn and regulates its degradation, implication for alpha-syn clearance and contribution to the pathogenesis of Parkinson's Disease. Hum Mol Genet 2014; 23: 2858-2879.

17. Cao $\mathrm{C}$, Leng $\mathrm{Y}$, Li $\mathrm{C}$, Kufe $\mathrm{D}$. Functional interaction between the $\mathrm{C}-\mathrm{Abl}$ and $\mathrm{Arg}$ protein-tyrosine kinases in the oxidative stress response. J Biol Chem 2003; 278: 12961-12967. 
18. Gotoh A, Miyazawa K, Ohyashiki K, Tauchi T, Boswell HS, Broxmeyer HE et al. Tyrosine phosphorylation and activation of focal adhesion kinase (p125FAK) by BCR-ABL oncoprotein. Exp Hematol 1995; 23: 1153-1159.

19. Sanguinetti AR, Mastick CC. C-Abl is required for oxidative stress-induced phosphorylation of caveolin-1 on tyrosine 14. Cell Signal 2003; 15: 289-298.

20. Burton EA, Oliver TN, Pendergast AM. Abl kinases regulate actin comet tail elongation via an N-WASP-dependent pathway. Mol Cell Biol 2005; 25: 8834-8843.

21. Rothweiler U, Aberg E, Johnson KA, Hansen TE, Jorgensen JB, Engh RA. p38alpha MAP kinase dimers with swapped activation segments and a novel catalytic loop conformation. J Mol Biol 2011; 411: 474-485.

22. Casanova E, Fehsenfeld S, Mantamadiotis T, Lemberger T, Greiner E, Stewart AF et al. A CamKIllalpha iCre BAC allows brain-specific gene inactivation. Genesis 2001; 31: 37-42.

23. Kohutnicka M, Lewandowska E, Kurkowska-Jastrzebska I, Czlonkowski A, Czlonkowska A. Microglial and astrocytic involvement in a murine model of Parkinson's disease induced by 1-methyl-4-phenyl-1,2,3,6-tetrahydropyridine (MPTP). Immunopharmacology 1998; 39: 167-180.

24. Hantschel O, Superti-Furga G. Regulation of the c-Abl and Bcr-Abl tyrosine kinases. Nat Rev Mol Cell Biol 2004; 5: 33-44.

25. Marley SB, Deininger MW, Davidson RJ, Goldman JM, Gordon MY. The tyrosine kinase inhibitor STI571, like interferon-alpha, preferentially reduces the capacity for amplification of granulocyte-macrophage progenitors from patients with chronic myeloid leukemia Exp Hematol 2000; 28: 551-557.

26. Schlatterer SD, Acker CM, Davies P. c-Abl in neurodegenerative disease. J Mol Neurosci 2011; 45: 445-452.

27. Johnston AM, Naselli G, Gonez LJ, Martin RM, Harrison LC, DeAizpurua HJ. SPAK, a STE20/SPS1-related kinase that activates the p38 pathway. Oncogene 2000; 19: 4290-4297.

28. Bi W, Xiao L, Jia $Y, W u J$, Xie $Q$, Ren J et al. c-Jun N-terminal kinase enhances MST1-mediated pro-apoptotic signaling through phosphorylation at serine 82. J Biol Chem 2010; 285: 6259-6264.

29. Zarubin T, Han J. Activation and signaling of the p38 MAP kinase pathway. Cell Res 2005; 15: 11-18.

30. Enslen H, Raingeaud J, Davis RJ. Selective activation of p38 mitogen-activated protein (MAP) kinase isoforms by the MAP kinase kinases MKK3 and MKK6. J Biol Chem 1998; 273: $1741-1748$.

31. Mittelstadt PR, Yamaguchi H, Appella E, Ashwell JD. T cell receptor-mediated activation of p38\{alpha\} by mono-phosphorylation of the activation loop results in altered substrate specificity. J Biol Chem 2009; 284: 15469-15474.

32. Jackson-Lewis V, Przedborski S. Protocol for the MPTP mouse model of Parkinson's disease. Nat Protoc 2007; 2: 141-151. 